\title{
Panorama do estado antropométrico dos escolares brasileiros
}

\author{
Anthropometric status of Brazilian schoolchildren
}

Caio Eduardo G. Reis ${ }^{1}$, Ivana Aragão L. Vasconcelos ${ }^{2}$, Odeth Maria V. Oliveira ${ }^{3}$

\section{RESUMO}

Objetivo: Revisar, na literatura, pesquisas que abordem o perfil antropométrico dos escolares brasileiros.

Fontes de dados: Buscaram-se artigos originais nas bases de dados SciELO, LILACS e MedLine, compreendidos no período entre 1985 e 2009, que avaliaram o estado antropométrico de escolares brasileiros (sete a dez anos). Foram utilizadas as palavras-chave: "escolar”, "escolares”, "avaliação nutricional”, “antropometria”, “criança”, “estatura”, “saúde escolar", em português e inglês.

Síntese dos dados: Foram encontrados 28 trabalhos realizados em escolas públicas e privadas de âmbito municipal, estadual e regional, com amostra variando de 86 a 50.114 indivíduos, com estudos do tipo transversal, inquérito, casoscontrole e prospectivo, utilizando seis tipos diferentes de protocolo para classificar o estado antropométrico dos escolares. Os resultados mostram que, nas regiões Norte, Nordeste e nas áreas carentes há maior prevalência de desnutrição, enquanto nas regiões Sudeste, Sul e zonas mais favorecidas há maior prevalência de sobrepeso e obesidade, mas coexistem as duas condições nas mesmas localidades.

Conclusões: Apesar do aumento ao acesso aos alimentos, das políticas públicas contra a fome e ações governamentais e não-governamentais, ainda há a coexistência da dupla carga de doenças, desnutrição e excesso de peso que aflige a população escolar brasileira. Ações simples como medidas de peso e altura são de suma importância para classificar o estado nutricional e definir rumos de políticas públicas.

Palavras-chave: escolares; avaliação nutricional; antropometria.

\section{ABSTRACT}

Objective: To review studies that address the anthropometric profile of Brazilian schoolchildren.

Data sources: Original articles published in SciELO, LILACS and MedLine databases from 1985 to 2009, which assessed the anthropometric status in Brazilian schoolchildren (seven to ten years old). Key-words used: "school", "schoolchild", "nutritional assessment", "anthropometry", "child", "stature”, "school health".

Data synthesis: Twenty eight publications were found. The studies were done in local and regional public and private schools, with samples ranging from 86 to 50,114 individuals. Cross-sectional, case-control, and prospective investigations were found, with six different types of protocol for the anthropometric status classification. The results show that there is a higher prevalence of malnutrition in the North and Northeast regions and in the deprived Brazilian areas, while in the Southeast and South regions there is a higher prevalence of overweight and obesity, but both conditions coexist in the same areas.

Conclusions: Despite the increased access to food, public policies against hunger and non-governmental and governmental actions, malnutrition and overweight still coexist in Brazilian schoolchildren. Simple actions such as measuring weight and height are extremely important for the nutritional status classification and for designing public policies.

Key-words: school children; nutrition assessment; anthropometry.
Instituição: Universidade de Brasília (UnB), Brasília, DF, Brasil

'Mestrando em Ciência da Nutrição pela Universidade Federal de Viçosa (UFV); Pesquisador do Laboratório de Metabolismo Energético e de Composição Corporal do Departamento de Nutrição e Saúde da UFV, Viçosa, MG, Brasil

2Doutoranda em Nutrição Humana pela UnB; Nutricionista da Coordenação de Atenção à Saúde do Servidor do Ministério da Saúde, Brasília, DF, Brasil Nutricionista da Secretaria de Estado de Saúde do Distrito Federal, Brasília, DF, Brasil

\author{
Endereço para correspondência: \\ Caio Eduardo G. Reis \\ Rua Pedro Gomide, 96, apto. 301 - Clélia Bernardes \\ CEP 36.570-000 - Viçosa/MG \\ E-mail: caioedureis@gmail.com
}

Conflito de interesse: nada a declarar

Recebido em: 22/9/2009

Aprovado em: 15/4/2010 


\section{Introdução}

Nas últimas décadas, as transformações socioeconômicas têm levado à transição nutricional, caracterizada por modificações no perfil nutricional da população brasileira. No passado, havia uma tendência de localização da prevalência de desnutrição em regiões menos favorecidas e bolsões de pobreza, e do excesso de peso em regiões ricas e desenvolvidas. Atualmente, pode-se encontrar ambas as condições em uma mesma localidade, e a sua relação com o perfil socioeconômico da população vem se tornando menos definida ${ }^{(1)}$.

A prevalência da desnutrição infantil está diminuindo com o passar dos anos, como foi demonstrado pelo Estudo Nacional sobre Despesas Familiares (Endef) ${ }^{(2)}$ e pela Pesquisa de Orçamentos Familiares $(\mathrm{POF})^{(3)}$, com redução de 16,6 para $4,6 \%$ entre 1975 e 2003. Já os casos de excesso de peso vêm aumentando (18,6 para $41 \%$ nos homens e 29,8 para 39,2\% nas mulheres), o que mostra uma tendência populacional ao ganho de peso. Os resultados da Pesquisa Nacional de Demografia e Saúde da Criança e da Mulber (PNDS) ${ }^{(4)}$, em 2006, mostram prevalência de $7 \%$ da desnutrição em crianças menores de cinco anos, sendo $15 \%$ na região Norte, e valores menores e similares nas demais regiões ( $6 \%$ nas regiões Centro-Oeste, Nordeste e Sudeste e $8 \%$ na região Sul). O excesso de peso esteve presente em $7 \%$ dos menores de cinco anos, variando de $6 \%$ na região Norte a $9 \%$ na região Sul.

Evidências indicam que a obesidade infantil tem crescido em torno de 10 a $40 \%$ na maioria dos países europeus nos últimos dez anos, apontando para uma epidemia mundial ${ }^{(5)}$. O excesso de peso na infância predispõe a várias complicações psicossociais, pois, muitas vezes, há isolamento social e afastamento das atividades devido à discriminação e à aceitação da criança diminuída pela sociedade. Em relação à saúde, a obesidade pode acarretar problemas respiratórios, diabetes melito, hipertensão arterial, dislipidemias, entre outros distúrbios ${ }^{(6)}$.

Nesse contexto de aumento do excesso de peso na população infantil, são necessários mais estudos que tracem um perfil nutricional dessa população, a fim de determinar com clareza a magnitude dessa problemática. O presente estudo teve como objetivo revisar estudos que abordam o perfil antropométrico dos escolares brasileiros.

\section{Métodos}

A pesquisa foi feita nas bases de dados eletrônicas Scientific Electronic Library Online Brasil (SciELO), Literatura
Latino-Americana e do Caribe em Ciências da Saúde (LILACS) e US National Libary of Medicine, Bethesda, MD (PubMed), sendo considerado período de publicação do artigo de 1985 até 2009. Foram empregadas as seguintes palavras-chave: "escolar(es)”, "avaliação nutricional”, "antropometria", "criança”, "estatura", "saúde escolar", suas combinações e seus respectivos termos em inglês. Dados adicionais foram extraídos das referências bibliográficas dos artigos encontrados. Para a seleção, os critérios de inclusão foram estudos com a população brasileira que avaliaram o estado antropométrico de escolares (sete a dez anos) e idades próximas. Foi feita uma avaliação dos critérios de inclusão pela leitura do resumo Os artigos selecionados foram classificados segundo o tema principal, o local de origem e a população estudada, sendo retiradas as seguintes informações: local e ano do estudo, ano de publicação, delineamento do estudo, caracterização da população, medida da avaliação antropométrica, métodos estatísticos e resultados encontrados. Foi pesquisado o Índice de Desenvolvimento Humano $(\mathrm{IDH})^{(7)}$ das localidades de coleta de dados das pesquisas, a fim de avaliar se as questões socioeconômicas interferem na questão antropométrica dessa população estudada.

\section{Resultados}

De acordo com os critérios de inclusão, 28 trabalhos estavam de acordo com o propósito da pesquisa ${ }^{(8-35)}$. O número de crianças pesquisadas em cada estudo variou de 86 a 50.114, mostrando uma grande heterogeneidade entre os trabalhos. Houve variação nos locais e no ambiente das pesquisas, que foram realizadas em escolas privadas e públicas de magnitude municipal, estadual e regional. Com relação à localidade, foi possível observar estudos de todas as regiões do país, com três na região Norte, oito na Nordeste, dois na Centro-Oeste, dez na Sudeste e cinco na Sul. Os estudos ocorreram em locais diversos e com populações diferentes, o que dificulta a comparação dos dados. As localidades variaram de assentamentos do movimento dos sem-terra, no interior de Alagoas, com IDH (2000) de 0.599, a Florianópolis, que tem o quarto maior IDH do Brasil (0.875), tendo o Brasil um IDH médio de 0.800 em $2000^{(7)}$. Notou-se também divergência no método da avaliação antropométrica utilizado.

A Tabela 1 mostra a caracterização dos artigos selecionados (autoria, ano de publicação, localidade/Estado, IDH, número amostral, idade, protocolo de avaliação antropométrica) e a Tabela 2 demonstra os pontos de corte utilizados nos estudos e os resultados principais. 
Tabela 1 - Caracterização dos estudos sobre o estado antropométrico dos escolares brasileiros

\begin{tabular}{|c|c|c|c|c|c|}
\hline $\begin{array}{l}\text { Autor, ano de } \\
\text { publicação }\end{array}$ & Localidade/Estado & $\mathrm{IDH}^{*}$ & $\mathbf{n}$ & $\begin{array}{l}\text { Idade } \\
\text { (anos) }\end{array}$ & $\begin{array}{l}\text { Protocolo de avaliação } \\
\text { antropométrica }\end{array}$ \\
\hline Neves, $2006^{(8)}$ & Belém/PA & 0,806 & 793 & 6 a 10 & OMS 1995 \\
\hline Orellana, $2006^{(9)}$ & $\begin{array}{l}\text { Reserva indígena } \\
\text { Suruí-Cacoal/RO }\end{array}$ & $0,729(a)$ & 284 & $<10$ & OMS 1995 \\
\hline Farias, $2008^{(10)}$ & Porto Velho/RO & 0,763 & 1.057 & 7 a 10 & $\begin{array}{l}\text { NCHS } 2000 \text { (P/E) e } \\
\text { Waterlow } 1977 \text { (P/E e E/l) }\end{array}$ \\
\hline Ferreira, $1997^{(11)}$ & Porto Calvo/AL & 0,599 & 357 & 1 a 10 & NCHS 1977 \\
\hline Carvalho, 2000(12) & Paraíba & 0,661 & 50.114 & 6 a 9 & NCHS 1977 \\
\hline Balaban, $2001^{(13)}$ & Recife/PE & 0,797 & 332 & 6 a 9 & OMS 1995 \\
\hline Alves, $2009^{(14)}$ & $\begin{array}{l}\text { Favelas do Fragoso e } \\
\text { Caranguejo, Recife/PE }\end{array}$ & $0,797(b)$ & 733 & 7 a 10 & NCHS 2000 \\
\hline Leão, 2003(15) & Salvador/BA & 0,805 & 387 & 5 a 10 & Himes 1994 e Anjos 1998 \\
\hline Silva, $2005^{(16)}$ & Maceió/AL & 0,739 & 1.253 & 7 a 17 & OMS 1995 \\
\hline Burlandy, 2007(17) & Nordeste e Sudeste & - & 1.177 & 7 a 10 & OMS 1995 \\
\hline Petroski, 2008 $8^{(18)}$ & Cotinguiba/SE & $0,635(c)$ & 480 & 7 a 9,9 & Cole 2000 \\
\hline Anjos, 1989(19) & Nova Iguaçu/RJ & 0,762 & 145 & 7 a 9,9 & NCHS 1977 \\
\hline Stefanini, $1995^{(20)}$ & Osasco/SP & 0,818 & 1.033 & 6 a 10 & NCHS, 1977 \\
\hline Malta, $1998^{(21)}$ & Belo Horizonte/MG & 0,839 & 699 & 6 a 7 & NCHS 1977 \\
\hline Veiga, $2001^{(22)}$ & Assentamento rural/RJ & $0,752(d)$ & 201 & 0 a 17 & $\begin{array}{l}\text { Anjos 1998; NCHS 1977; } \\
\text { OMS } 1995\end{array}$ \\
\hline Sotelo, $2004^{(23)}$ & São Paulo/SP & 0,841 & 2.509 & 6 a 11 & $\begin{array}{l}\text { OMS 1995; Cole } 2000 \text { e } \\
\text { Must1991 }\end{array}$ \\
\hline Costa, $2006^{(24)}$ & Santos/SP & 0,871 & 10.822 & 7 a 10 & NCHS 2000 \\
\hline Mondini, $2007^{(25)}$ & Cajamar/SP & 0,786 & 1.014 & 6 a 7 & Cole 2000 \\
\hline Santos, $2007^{(26)}$ & São Paulo/SP & 0,841 & 86 & 7 a 15 & Himes 1994 \\
\hline Fagundes, $2008^{(27)}$ & São Paulo/SP & 0,841 & 218 & 6 a 14 & OMS 2006 \\
\hline Pegolo, $2008^{(28)}$ & Piedade/SP & 0,757 & 150 & 7 a 14 & NCHS 2000 \\
\hline Giugliano, 2004(29) & Taguatinga/DF & 0,844 & 452 & 6 a 10 & Cole 2000 \\
\hline Guimarães, 2006(30) & Cuiabá/MT & 0,821 & 1.256 & 6 a 11 & OMS, 1995 \\
\hline Corso, 2001(31) & Florianópolis/SC & 0,875 & 4.591 & 6 a 8 & OMS 1995 \\
\hline Soar, 2004(32) & Florianópolis/SC & 0,875 & 419 & 7 a 9 & Cole 2000 \\
\hline Ricardo, 2009(33) & Estado de SC & 0,875 & 4.964 & 6 a 10 & Cole 2000 \\
\hline Ronque, $2005^{(34)}$ & Londrina/PR & 0,824 & 511 & 7 a 10 & NCHS 2000 \\
\hline Triches, $2005^{(35)}$ & $\begin{array}{l}\text { Dois Irmãos/RS; } \\
\text { Morro Reuter/RS }\end{array}$ & $\begin{array}{l}0,812 \\
0,834\end{array}$ & 573 & 8 a 11 & NCHS 1977 \\
\hline
\end{tabular}

* Fonte do Índice de Desenvolvimento Humano (IDH): Programa das Nações Unidas para o Desenvolvimento, 2000(7); (a) Média do IDH dos municípios Cacoal, no Estado de Rondônia, e Aripuanã, no Estado de Mato Grosso; (b) Área sem avaliação do IDH, dado aproximado da região metropolitana de Recife; (c) Média do IDH dos municípios Capela, Divina Pastora, Santa Rosa de Lima e Siriri, que compõem a mesorregião; (d) Área sem avaliação do IDH, dado aproximado do município Cachoeiras de Macacu. 
Tabela 2 - Resultados dos estudos sobre o estado antropométrico dos escolares brasileiros

\begin{tabular}{|c|c|c|}
\hline $\begin{array}{l}\text { Autor, ano de } \\
\text { publicação }\end{array}$ & Pontos de corte descritos pelos estudos (a) & Resultados (b) \\
\hline \multirow[t]{3}{*}{ Neves, $2006^{(8)}$} & Baixa estatura (E/I): $Z<-2$ e Risco: $-2 \leq Z \leq-1,5$ & $16,6 \%$ BE e RBE \\
\hline & Baixo peso (P/I): $Z<-2$ e Risco: $-2 \leq Z \leq-1,5$ & $4,5 \%$ BP e RBP \\
\hline & Sobrepeso (P/l): $+1,5 \leq \mathrm{Z} \leq+2$ e Obesidade (P/I): Z>+2 & $7,4 \%$ SP e OBS \\
\hline \multirow[t]{2}{*}{ Orellana, $2006^{(9)}$} & Baixo peso $(\mathrm{P} / \mathrm{l})$ : $\mathrm{Z}<-2$ & $25,4 \%$ BP \\
\hline & Baixa estatura (E/I): Z<-2 & $8,1 \% \mathrm{BE}$ \\
\hline \multirow[t]{4}{*}{ Farias, $2008^{(10)}$} & Desnutrição crônica: $P / E$ e E/I $\leq-2$ & $1,5 \% \mathrm{DC}$ \\
\hline & Desnutrição pregressa: $P / E>-2$ e $E / I \leq-2$ & $7,0 \% \mathrm{DP}$ \\
\hline & Desnutrição aguda: $P / E \leq-2$ e $E / l \geq-2$ & $2,5 \% \mathrm{DA}$ \\
\hline & Eutrofia: P/E e E/I com Z>-2 & $89 \%$ Eut \\
\hline \multirow[t]{2}{*}{ Ferreira, $1997^{(11)}$} & Baixo peso (P/I): Z<-2 & $47,1 \% \mathrm{BP}$ \\
\hline & Baixa estatura (E/I): Z<-2 & $41,2 \% \mathrm{BE}$ \\
\hline \multirow[t]{2}{*}{ Carvalho, $2000^{(12)}$} & Baixa estatura (E/I): $Z<-2$ & $17,8 \%$ BE área rural \\
\hline & & $11,8 \%$ BE área urbana \\
\hline \multirow[t]{2}{*}{ Balaban, $2001^{(13)}$} & Sobrepeso: $I M C \geq 85$ th para idade e sexo & Meninos: $33,6 \%$ SP; $19,7 \%$ OBS \\
\hline & Obesidade: IMC e prega triciptal $\geq 85$ th & Meninas: $34,3 \%$ SP; $14,2 \%$ OBS \\
\hline \multirow[t]{2}{*}{ Alves, 2009(14) } & Sobrepeso: IMC>85th & $12,6 \% \mathrm{SP}+\mathrm{OBS}$ \\
\hline & Obesidade: IMC>95th & \\
\hline \multirow[t]{2}{*}{ Leão, $2003^{(15)}$} & Obesidade: IMC $\geq 95$ th & $30 \%$ OBS - escolas particulares \\
\hline & & 8\% OBS - escolas públicas \\
\hline \multirow[t]{2}{*}{ Silva, $2005^{(16)}$} & Risco de sobrepeso: 85 th $\leq I M C<95$ th & 9,25 Risco de SP \\
\hline & Sobrepeso: IMC $\geq 95$ th & $4,5 \% \mathrm{SP}$ \\
\hline \multirow[t]{2}{*}{ Burlandy, 2007(17) } & Baixo peso: $Z<-2$ & $13,2 \%$ BP \\
\hline & Sobrepeso: Z>+2 & $9,5 \% \mathrm{SP}$ \\
\hline \multirow[t]{2}{*}{ Petroski, 2008(18) } & Desnutrição: IMC 16-18,5kg/m² & $22,7 \% \mathrm{BP}$ \\
\hline & Excesso de peso: $I M C \geq 25 \mathrm{~kg} / \mathrm{m}^{2}$ & $5,4 \% \mathrm{SP}+\mathrm{OBS}$ \\
\hline \multirow[t]{2}{*}{ Anjos, 1989(19) } & Desnutrição (P/E e E/l): Z<-2 & 4,9\% Desnutrição \\
\hline & Obesidade (P/E): Z>+2 & $23,15 \%$ OBS \\
\hline Stefanini, $1995^{(20)}$ & Baixa estatura (E/I): $Z<-2$ & $3,9 \% \mathrm{BE}$ \\
\hline \multirow[t]{4}{*}{ Malta, $1998^{(21)}$} & Baixa estatura $(E / I):<2,3$ th & $7,2 \% \mathrm{BE}$ \\
\hline & Risco para baixa estatura (E/I): 2,31-10th & $19,5 \%$ RBE \\
\hline & Baixo peso $(\mathrm{P} / \mathrm{I})$ : $<2,3$ th & $3,4 \% \mathrm{BP}$ \\
\hline & Risco de Baixo peso $(\mathrm{P} / \mathrm{l})$ : 2,31-10th & $12,9 \%$ RBP \\
\hline \multirow[t]{3}{*}{ Veiga, 2001(22) } & Baixa estatura (E/I): Z<-2 & $1,6 \% \mathrm{BE}$ \\
\hline & Baixo peso (P/E): Z<-2 & $1,5 \% \mathrm{BP}$ \\
\hline & Sobrepeso (P/E): Z<+2 & $3,1 \% \mathrm{SP}$ \\
\hline
\end{tabular}


Continuação da Tabela 2.

\begin{tabular}{|c|c|c|}
\hline $\begin{array}{l}\text { Autor, ano de } \\
\text { publicação }\end{array}$ & Pontos de corte descritos pelos estudos (a) & Resultados (b) \\
\hline \multirow[t]{2}{*}{ Sotelo, $2004^{(23)}$} & Sobrepeso (P/E): +1<Zs+2 e IMC/I 85th-95th & $\begin{array}{l}\text { Meninos: } 10,3 \text { e } 11,9 \% \text { SP; } 8,2 \text { e } \\
13,7 \% \text { OBS (c) }\end{array}$ \\
\hline & Obesidade (P/E): Z>+2 e IMC/I>95th & $\begin{array}{l}\text { Meninas: } 13,5 \text { e } 13,8 \% \text { SP; } 8,2 \text { e } \\
16,5 \% \text { OBS (c) }\end{array}$ \\
\hline \multirow[t]{2}{*}{ Costa, $2006^{(24)}$} & Sobrepeso: 85 th $\leq 1 \mathrm{MC} \leq 95$ th & $15,7 \% \mathrm{SP}$ \\
\hline & Obesidade: IMC $\geq 95$ th & $18 \%$ OBS \\
\hline \multirow[t]{2}{*}{ Mondini, $2007^{(25)}$} & Sobrepeso: IMC/I 85th-95th & $10,8 \% \mathrm{SP}$ \\
\hline & Obesidade: IMC/I>95th & $6,2 \%$ OBS \\
\hline \multirow[t]{3}{*}{ Santos, $2007^{(26)}$} & Baixo peso $(P / I): Z<-2$ & Meninos: 5,6\% BE; 5,6\% BP; $11,2 \%$ SP \\
\hline & Baixa estatura $(E / I): Z<-2$ & Meninas: $8 \%$ BE; $2 \%$ BP; $30 \%$ SP \\
\hline & Sobrepeso: Z>+2 & \\
\hline \multirow[t]{4}{*}{ Fagundes, $2008^{(27)}$} & Baixo peso: $P / E<95 \%$ & $25,7 \% \mathrm{BP}$ \\
\hline & Eutrofia: $95 \%<P / E<110 \%$ & $43,1 \%$ Eut \\
\hline & Sobrepeso: $110 \%<P / E<120 \%$ & $16,5 \% \mathrm{SP}$ \\
\hline & Obesidade: P/E>120\% & $14,7 \%$ OBS \\
\hline \multirow[t]{4}{*}{ Pegolo, $2008{ }^{(28)}$} & Baixa estatura $(\mathrm{A} / \mathrm{l})$ : $\mathrm{Z}<-2$ & $4,0 \% \mathrm{BE}$ \\
\hline & Risco de Baixa estatura $(A / I):-2 \leq Z<-1$ & $19,3 \%$ RBE \\
\hline & Baixo peso: IMC $\leq 5$ th & $10,7 \% \mathrm{BP}$ \\
\hline & Obesidade: IMC $\geq 95$ th & $6,6 \%$ OBS \\
\hline \multirow[t]{3}{*}{ Giugliano, 2004(29) } & Baixo Peso: IMC/I<5th & $3,1 \% \mathrm{BP}$ \\
\hline & Sobrepeso: IMC/I 85th-95th & $16,8 \% \mathrm{SP}$ \\
\hline & Obesidade: IMC/I>95th & $5,3 \%$ OBS \\
\hline \multirow[t]{2}{*}{ Guimarães, 2006(30) } & Sobrepeso: IMC/I $\geq 85$ th & $7,75 \%$ SP \\
\hline & Obesidade: IMC/I $\geq 95$ th & $6,65 \%$ OBS \\
\hline Corso, $2001^{(31)}$ & Baixa estatura $(\mathrm{A} / \mathrm{l})$ : $Z<-2$ & $3,1 \% \mathrm{BE}$ \\
\hline \multirow[t]{2}{*}{ Soar, 2004(32) } & Sobrepeso: IMC $\geq 18,03$ a $19,46 \mathrm{~kg} / \mathrm{m}^{2}$ (d) & $17,9 \% \mathrm{SP}$ \\
\hline & Obesidade: IMC $\geq 21,01$ a $23,46 \mathrm{~kg} / \mathrm{m}^{2}$ (d) & $6,7 \%$ OBS \\
\hline \multirow[t]{2}{*}{ Ricardo, $2009^{(33)}$} & Sobrepeso: IMC>25 kg/m² e $<30 \mathrm{~kg} / \mathrm{m}^{2}$ & $15,4 \% \mathrm{SP}$ \\
\hline & Obesidade IMC $\geq 30$ kg/m² & $5,4 \%$ OBS \\
\hline \multirow[t]{2}{*}{ Ronque, $2005^{(34)}$} & Sobrepeso: 85 th $\leq I M C \leq 95$ th & Meninos: $19,7 \%$ SP e $17,5 \%$ OBS \\
\hline & Obesidade: IMC $\geq 95$ th & Meninas: $17,3 \%$ SP e $9,3 \%$ OBS \\
\hline \multirow[t]{2}{*}{ Triches, $2005^{(35)}$} & Sobrepeso: IMC>85th & Meninos: $17,3 \%$ SP e $7,4 \%$ OBS \\
\hline & Obesidade: IMC>95th & Meninas: $16,6 \%$ SP e $7,6 \%$ OBS \\
\hline
\end{tabular}

* Fonte do Índice de Desenvolvimento Humano (IDH): Programa das Nações Unidas para o Desenvolvimento, 2000(7); (a) IMC/I: índice de massa corpórea por idade; P/l: peso por idade; E/l: estatura por idade; P/E: peso por estatura; (b) BP (baixo peso), RBP (risco para baixo peso), Eut (eutrofia), SP (sobrepeso), OBS (obesidade), BE (baixa estatura), RBE (risco para baixa estatura), DA (desnutrição aguda), DP (desnutrição pregressa), DC (desnutrição crônica); (c) A depender do ponto de corte; (d) A depender do sexo e idade. 


\section{Discussão}

Cerca de $7 \%$ da população infantil mundial é obesa, e duas a três vezes mais crianças estão com sobrepeso ${ }^{(36)}$. As maiores taxas de obesidade infantil têm sido observadas em países desenvolvidos, mas a prevalência está cada vez mais elevada nos países em desenvolvimento. Estimativas do ano 2000 sugerem que os países desenvolvidos têm cerca de 10 a $25 \%$ de sua população infantil acima do peso. Tais dados são alarmantes, pois crianças com excesso de peso tendem a ser adultos obesos ${ }^{(37)}$. Os dados do Brasil são semelhantes aos da América Latina, que está passando por uma rápida transição demográfica e nutricional, com declínio das mortes por infecção e aumento do excesso de peso ${ }^{(38)}$.

$\mathrm{Na}$ região Norte, os trabalhos de Neves et al ${ }^{(8)}$ e Orellana et $a l^{(9)}$ são similares metodologicamente: ambos são de corte transversal e utilizam os padrões de referência para classificação do estado antropométrico os valores da Organização Mundial da Saúde (OMS - 1995), facilitando a comparação dos resultados. É nítida a presença da desnutrição nas localidades, sendo a desnutrição mais grave na reserva indígena. Belém passa pelo fenômeno da dupla carga de doenças, convivendo simultaneamente com os problemas da desnutrição e da obesidade. Os artigos mostram a realidade da região: Belém, uma metrópole da Amazônia, e uma comunidade indígena (localizada nos municípios de Cacoal, no Estado de Rondônia, e Aripuanã, no Estado de Mato Grosso). Farias, em um estudo de base populacional $(\mathrm{n}=1.057)$ de Porto Velho, Rondônia, mostrou a prevalência de desnutrição de $11 \%$ nos alunos de baixo nível socioeconômico de uma escola municipal da cidade em $2008^{(10)}$. Os resultados exibem a realidade carencial encontrada na região e indicam a necessidade de aumento de ações públicas e privadas para modificar esses dados. Há um baixo número de publicações com essa população, possivelmente devido ao difícil acesso às localidades e à falta de recursos humanos para tal função.

$\mathrm{Na}$ região Nordeste, os estudos têm critérios de classificação do estado antropométrico diferentes, mas é possível constatar que os índices de desnutrição não são baixos. Petroski et al, em $2008^{(18)}$, publicaram os resultados da pesquisa realizada na região de Cotinguiba (microrregião do estado de Sergipe pertencente à mesorregião do Leste Sergipano), que está dividida em quatro municípios: Capela, Divina Pastora, Santa Rosa de Lima e Siriri, encontrando resultados expressivos: $22,7 \%$ de baixo peso e $5,4 \%$ de sobrepeso e obesidade. Já Silva et al ${ }^{(16)}$, após uma avaliação dos escolares do município de Maceió, observaram 4,5\% de sobrepeso, em geral nas crianças que frequentavam escolas privadas. O estudo realizado em Salvador por Leão $e t$ al $l^{(15)}$, em 2000, mostra prevalência de obesidade em escolares de $30 \%$ em escolas particulares e $8 \%$ em públicas. Já Balaban e Silva ${ }^{(13)}$ publicaram os resultados de um estudo realizado em Recife, também preocupantes: 33,6 e 34,3\% de sobrepeso e 19,7 e $14,2 \%$ de obesidade nos meninos e meninas, respectivamente. Salvador e Recife foram os locais com o IDH mais alto $(0,805$ e 0,797 , respectivamente) e com as maiores porcentagens de excesso de peso dentre os estudos provenientes da região Nordeste. Ferreira $e t a^{(11)}$ avaliaram crianças residentes no acampamento do movimento dos semterra no município de Maceió (IDH 0,599) e observaram alta prevalência de desnutrição ( $47,1 \%$ de baixo peso). Esses resultados indicam que o nível socioeconômico e a condição de desenvolvimento influenciam no estado nutricional das crianças, com maior prevalência de desnutrição nos locais mais pobres e, em contrapartida, nos mais favorecidos, maior prevalência de sobrepeso e obesidade.

Porém, a relação entre nível socioeconômico ou desenvolvimento e estado nutricional nem sempre segue essa lógica. Exemplo disso é o estudo de Alves et a ${ }^{(14)}$, realizado nas favelas do Fragoso e Caranguejo, Recife, PE, com 733 escolares, publicado em 2009. Os autores notaram uma frequência de excesso de peso de 12,6\%, relacionada à inatividade física. Ou seja, mesmo em localidades carentes, é possível observar diferenças no padrão nutricional dos escolares, refletindo a transição nutricional e a coexistência de desnutrição e obesidade. Nesses casos, a situação pode se tornar mais grave por preocupação maior dessas famílias mais carentes com a desnutrição do que com os casos de sobrepeso e obesidade, chegando a negligenciá-los.

Estudos mais robustos vêm contribuindo com informações importantes. Burlandy et al ${ }^{(17)}$ publicaram o resultado de uma pesquisa multicêntrica a respeito da avaliação do estado nutricional de escolares do Nordeste e Sudeste do Brasil, por meio da análise dos dados da Pesquisa sobre Padrões de Vida. Foram avaliadas 1.177 crianças de sete a dez anos entre 1995 e 1997. A desnutrição ocorreu em 13,2\% das crianças e foi mais prevalente na região rural do Nordeste em relação à região urbana do Sudeste. A prevalência de sobrepeso foi relativamente alta $(9,5 \%)$ e similar para meninos e meninas. Já Carvalho $e t$ al ${ }^{(12)}$, ao fazerem uma avaliação de mais de 50 mil crianças no Estado da Paraíba (IDH 0,661), observaram prevalência de $17,8 \%$ de baixa estatura na zona rural e 11,8\% na zona urbana. Dos que apresentaram baixa estatura, $18,7 \%$ 
encontravam-se na região do sertão, 13,8\% no agreste, 11,9\% na zona do Borborema e $10,9 \%$ na zona da mata.

A garantia do direito humano à alimentação adequada por meio da segurança alimentar e nutricional é lei federal desde 2006 (lei n. ${ }^{\circ} 11.346$ ). Essa legislação deve garantir que todo brasileiro tenha o direito a uma alimentação permanente, regular e adequada do ponto de vista quantitativo e qualitativo, sem que isso comprometa outras necessidades básicas. Esse acesso deve ter como pilar as práticas alimentares promotoras da saúde, de forma a respeitar a diversidade cultural e abordar todas as suas dimensões de maneira sustentável. Esse direito não será plenamente cumprido se a qualidade do alimento estiver comprometida, mesmo que a disponibilidade de alimentos seja suficiente e a população tenha acesso a eles. O extremo da violação desse direito à alimentação, ou seja, a fome, é inaceitável pelo Pacto Internacional sobre os Direitos Econômicos, Sociais e Culturais ${ }^{(39,40)}$.

$\mathrm{Na}$ região Sudeste, Stefanini et a ${ }^{(20)}$ mostram prevalência de baixa estatura de 3,97\%, Malta et al ${ }^{(21)}$ e Pegolo et al ${ }^{(28)}$ detectam 7,2 e 4,0\% dos escolares com baixa estatura, e 3,4 e 10,7\% dos alunos com baixo peso, respectivamente. As três pesquisas avaliaram alunos de escolas públicas, utilizando como referência os padrões do NCHS (1977 e 2000). O estudo de Veiga e Burlandy ${ }^{(22)}$, realizado em um assentamento rural no Estado do Rio de Janeiro (IDH 0,752), mostra a prevalência de 1,6\% de baixa estatura e 3,2\% baixo peso. Anjos $^{(19)}$ avaliou o estado nutricional de escolares de baixa renda de um município do Estado do Rio de Janeiro e obteve resultados semelhantes: $3,52 \%$ das crianças desnutridas e 6,25\% com desnutrição crônica, dados que se comparam aos de favelas do município do Rio de Janeiro. Nesses estudos, os resultados corroboram a influência do nível social na desnutrição.

Os trabalhos de Sotelo et al ${ }^{(23)}$, Costa $e t a^{(24)}$, Mondini et $a l^{(25)}$, Santos et $a l^{(26)}$ e Fagundes $e t a l^{(27)}$ foram realizados no Estado de São Paulo e relatam um alto nível de sobrepeso (16\%) e obesidade (18\%). Quando avaliadas as crianças com excesso de peso, essa prevalência sobe para mais de $20 \%$, o que pode ser explicado, em parte, pela urbanização e industrialização da sociedade moderna. Esse contexto contribui para mudanças no estilo de vida da população, como o aumento no consumo de produtos gordurosos e ricos em açúcar ${ }^{(41)}$ e concomitante redução dos níveis de atividade física ${ }^{(42)}$.

No Centro-Oeste, os dois trabalhos encontrados realizados em Taguatinga, DF (2004) ${ }^{(29)}$, e Cuiabá, MT (2006) ${ }^{(30)}$, com 0,844 e 0,822 de IDH, respectivamente, avaliaram crianças de faixas etárias próximas (seis a dez anos e seis a 11 anos). Seus resultados indicam uma prevalência de sobrepeso elevada (16,8\% em Taguatinga, DF, e 14,4\% em Cuiabá, MT). A adiposidade associou-se ao sedentarismo e foi maior em escolares com renda familiar per capita maior que três salários mínimos e com atividades físicas com carga horária inferior a dez horas por semana. De acordo com o recente estudo publicado por Dunstan et al ${ }^{(43)}$, parece haver também associação entre o sedentarismo e o risco elevado de desenvolver doenças cardiovasculares. Uma vez que a obesidade está relacionada às maneiras de viver e às condições de vida, as estratégias de prevenção e controle sugeridas pelo Ministério da Saúde no âmbito populacional compreendem, além da abordagem educacional, medidas que contemplam as diversas dimensões do ambiente - física, econômica, política e sociocultural - a fim de torná-lo menos obesogênico ${ }^{(44)}$. Sendo assim, facilitar o acesso a frutas e hortaliças para as populações de baixa renda e propiciar espaços de lazer seguros para a prática de atividade física seriam exemplos de estratégias para criar ambientes mais saudáveis.

Programas de educação nutricional e políticas públicas de saúde vêm sendo desenvolvidos com o objetivo de promover práticas alimentares saudáveis no ambiente escolar, a fim de prevenir agravos à saúde ${ }^{(45,46)}$. Por meio desses programas, é possível instruir e mobilizar crianças, jovens, famílias e comunidades quanto à adoção de hábitos saudáveis e a criação de ambientes saudáveis, como cantinas escolares, horta escolar e comunitária e espaços para prática de exercício físico, que trarão melhor qualidade de vida à população ${ }^{(47)}$.

Finalmente, na região Sul, foram encontrados três estudos realizados em Florianópolis, Santa Catarina. Corso et al ${ }^{(31)}$, avaliando escolares de famílias carentes $(n=4.591)$, revelaram o perfil do estado nutricional com uma taxa de baixa estatura de 3,1\%. Já Soar et al ${ }^{(32)}$ estudaram alunos de uma escola pública localizada na região central do município e observaram $17,9 \%$ de sobrepeso e $6,7 \%$ de obesidade. Ao separarem os escolares por áreas, verificaram que apenas 2,3\% dos residentes em áreas não carentes apresentaram desnutrição por déficit estatural, enquanto $4,1 \%$ de áreas carentes apresentaram baixa estatura, enfatizando a associação da condição socioeconômica ao estado nutricional. Em estudo recente publicado por Ricardo et al ${ }^{(33)}$, foi traçado o perfil antropométrico dos escolares do Estado de Santa Catarina, com uma amostra probabilística de 4.964 alunos de seis a dez anos matriculados em 345 escolas públicas e privadas das três grandes regiões do Estado (Oeste, Centro e Litoral). Sua amostra foi composta por $52,2 \%$ de meninas, 
tendo como resultado $15,4 \%$ de sobrepeso e 5,4\% de obesidade. A prevalência de sobrepeso foi de 19,7\% nos alunos de escolas particulares e de 14,3\% nos de escola pública, tendo os alunos da rede particular 1,46 (1,22-1,74) vezes mais chance de desenvolverem sobrepeso do que os da rede pública. Os trabalhos apresentados revelam a alta prevalência de excesso de peso na população estudada no estado de Santa Catarina, sendo de grande importância que a Saúde Pública do Estado aborde de forma apropriada o problema. Já no Paraná, Ronque et al ${ }^{(34)}$, ao avaliarem 511 escolares de Londrina (IDH 0,824), detectaram uma prevalência de 19,7 e $17,3 \%$ de sobrepeso e 17,5 e $9,3 \%$ de obesidade em meninos e meninas, respectivamente. No Rio Grande do Sul, Triches e Giugliani ${ }^{(35)}$ avaliaram dois municípios gaúchos e encontraram resultados semelhantes aos de Florianópolis, com prevalência de sobrepeso e obesidade no sexo feminino de 16,6 e 7,6\%, respectivamente, e no sexo masculino de 17,3 e $7,4 \%$. A obesidade mostrou-se associada a práticas alimentares menos saudáveis.

\section{Considerações finais}

A transição nutricional na população brasileira é caracterizada pela redução da prevalência do déficit nutricional e aumento na ocorrência do excesso de peso. A população infantil vem passando por uma transformação em seus hábitos alimentares e de vida que afetam a composição corporal e as condições de saúde. Campanhas para a promoção da saúde, no contexto de hábitos alimentares e práticas de atividades físicas, são importantes para incentivar o estilo de vida saudável por todas as camadas da população. O conhecimento dos fatores de risco e da distribuição da desnutrição e da obesidade infantil se faz necessário para definir prioridades e estratégias de ações em Saúde Pública, especialmente na prevenção e no controle dessas morbidades, diminuindo o risco de essas crianças se tornarem adultos obesos.

\section{Referências bibliográficas}

1. Batista Filho M, Rissin A. Nutritional transition in Brazil: geographic and temporal trends. Cad Saude Publica 2003;19:S181-91.

2. Instituto Brasileiro de Geografia e Estatística - IBGE. Estudo nacional da despesa familiar - ENDEF: dados preliminares - consumo alimentar antropometria. Rio de Janeiro: IBGE; 1977.

3. Instituto Brasileiro de Geografia e Estatística - IBGE. Pesquisa de Orçamentos Familiares 2002-2003: antropometria e análise do estado nutricional de
Nesse contexto, novas políticas públicas em relação aos cuidados alimentares e nutricionais com as crianças devem ser elaboradas. Para embasar essas políticas, se faz necessário o diagnóstico do estado nutricional da população brasileira de escolares, já que não há uma pesquisa nacional que aponte a prevalência dos agravos para essa faixa etária. Em março de 2009, o Instituto Brasileiro de Geografia e Estatística (IBGE) iniciou a coleta de dados da Pesquisa Nacional de Saúde do Escolar (PeNSE), que pretende avaliar 70 mil alunos de 1.507 escolas públicas e privadas de todo o país, com questões de alimentação, atividade física, condições de vida e violência, entre outras. Os estudantes também serão pesados e medidos. Porém, é um estudo que englobará somente adolescentes de 13 a 15 anos $\left(8^{a} \text { série ou } 9^{\circ} \text { ano }\right)^{(48)}$.

Uma parceria permanente entre serviços de saúde e escolas é uma estratégia apresentada pelo Ministério de Saúde para permitir que a vigilância alimentar/nutricional dos escolares seja realizada e fortalecida no país. Propõe-se que o acompanhamento dos escolares pelo Sistema de Vigilância Alimentar e Nutricional (SISVAN), com o objetivo de fornecer informação sobre o perfil alimentar e nutricional e os fatores que o influenciam, seja feito no mínimo uma vez por ano, dependendo de como o sistema está implantado na localidade ${ }^{(49)}$. Se assim for feito, essa faixa etária da população terá o diagnóstico traçado periodicamente, o que ajudará a formular e avaliar as políticas públicas.

Os estudos aqui apresentados relatam o panorama do estado nutricional dos escolares de várias localidades do Brasil, que, apesar do aumento ao acesso aos alimentos, das políticas públicas contra a fome e demais ações, ainda contam com a dupla carga de agravos nutricionais (desnutrição e excesso de peso). São necessários novos estudos com grandes populações para que se trace um perfil nutricional completo da população infantil, o que poderá fornecer subsídios para programas de intervenção em promoção de saúde e ações na prevenção e redução das prevalências de sobrepeso e obesidade em escolares. crianças e adolescentes no Brasil. Rio de Janeiro: IBGE; 2004.

4. Ministério da Saúde - MS. Pesquisa nacional de demografia e saúde da criança e da mulher. Brasília: Ministério da Saúde; 2008.

5. Dietz WH. The obesity epidemic in young children. Reduce television viewing and promote playing. BMJ 2001;322:313-4.

6. Ebbeling CB, Pawlak DB, Ludwig DS. Childhood obesity: public-health crisis, common sense cure. Lancet 2002;360:473-82. 
7. Programa das Nações Unidas para o Desenvolvimento, 2000 [homepage on the Internet]. Índice de desenvolvimento humano, municipal $1991-2000$ [cited 2010 Jan 19]. Available from: http://www.pnud.org.br/atlas/ranking/ IDH-M\%2091\%2000\%20Ranking\%20decrescente\%20(pelos $\% 20$ dados $\% 20$ de\%202000).htm

8. Neves OM, Brasil AL, Brasil LM, Taddei JA. School aged anthropometry when enrolling in the first grade of elementary school in the city of Belém, Pará, 2001. Rev Bras Saúde Matern Infant 2006;6:39-46.

9. Orellana JD, Coimbra Jr CE, Lourenço AE, Santos RV. Estado nutricional e anemia em crianças Suruí, Amazônia, Brasil. J Pediatr (Rio J) 2006;82:383-8.

10. Farias ES, Guerra-Júnior G, Petroski EL. Estado nutricional de escolares em Porto Velho, Rondônia. Rev Nutr 2008;21:401-9.

11. Ferreira HS, Albuquerque MF, Ataide TR, Morais MG, Mendes MC, Siqueira TC et al. Nutritional status of children ten years of age or under in a settlement organized by the "Landless Peasants' Movement" in Porto Calvo, Alagoas, Brazil. Cad Saude Publica 1997;13:137-9.

12. Carvalho AT, Costa MJ, Ferreira LO, Batista Filho M. Cartografia do retardo estatural em escolares do Estado da Paraíba, Brasil. Rev Saude Publica 2000;34:3-8.

13. Balaban G, Silva GA. Overweight and obesity prevalence in children and adolescents from a private school in Recife. J Pediatr (Rio J) 2001;77:96-100.

14. Alves JG, Siqueira PP, Figueiroa JN. Overweight and physical inactivity in children living in favelas in the metropolitan region of Recife, Brazil. J Pediatr (Rio J) 2009;85:67-71.

15. Leão LS, Araújo LM, Moraes LT, Assis AM. Prevalência de obesidade em escolares de Salvador, Bahia. Arq Bras Endocrinol Metab 2003;47:151-7.

16. Silva MA, Rivera IR, Ferraz MR, Pinheiro AJ, Alves SW, Moura AA et al. Prevalence of cardiovascular risk factors in child and adolescent students in the city of Maceió. Arq Bras Cardiol 2005;84:387-92.

17. Burlandy L, dos Anjos LA. Access to the school food program and nutritional status of schoolchildren in Northeast and Southeast Brazil, 1997. Cad Saude Publica 2007;23:1217-26.

18. Petroski EL, Silva RJ, Pelegrini A. Crescimento físico e estado nutricional de crianças e adolescentes da região de Cotinguiba, Sergipe. Rev Paul Pediatr 2008;26:206-11.

19. dos Anjos LA. Índices antropométricos e estado nutricional de escolares de baixa renda de um município do Estado do Rio de Janeiro (Brasil): um estudo piloto. Rev Saude Publica 1989;23:221-9.

20. Stefanini ML, Colli C, Lerner BR, Lei DL, Chaves SP, di Pietro MS et al. Anemia and malnutrition in children at public schools in Osasco, São Paulo, Brazil. Cad Saude Publica 1995;11:439-47.

21. Malta DC, Goulart EM, Lima e Costa MF. Nutritional status and socioeconomic factors associated with failure in school: a prospective study of first grade students in Belo Horizonte, Brazil. Cad Saude Publica1998;14:157-64.

22. Veiga GV, Burlandy L. Socioeconomic and demographic indicators and nutritional status of children in a rural land settlement in Rio de Janeiro. Cad Saude Publica 2001;17:1465-72.

23. Sotelo Yde O, Colugnati FA, Taddei JA. Prevalence of overweight and obesity in public school pupils according to three anthropometric diagnostic criteria. Cad Saude Publica 2004;20:233-40.

24. Costa RF, Cintra IP, Fisberg M. Prevalência de sobrepeso e obesidade em escolares da cidade de Santos, SP. Arq Bras Endocrinol Metab 2006;50:60-7.

25. Mondini L, Levy RB, Saldiva SR, Venâncio SI, de Azevedo Aguiar J, Stefanini ML. Overweight, obesity and associated factors in first grade schoolchildren in a city of the metropolitan region of São Paulo, Brazil. Cad Saude Publica 2007;23:1825-34.

26. Santos EB, Amâncio OM, Oliva CA. Estado nutricional, ferro, cobre e zinco em escolares de favelas da cidade de São Paulo. Rev Assoc Med Bras 2007;53:323-8.

27. Fagundes AL, Ribeiro DC, Naspitz L, Garbelini LE, Vieira JK, Silva AP et al. Prevalence of overweight and obesity in school children of Parelheiros region in São Paulo city, Brazil. Rev Paul Pediatr 2008;26:212-7.
28. Enes CC, Pegolo GE, Silva MV. Influência do consumo alimentar e do padrão de atividade física sobre o estado nutricional de adolescentes de Piedade, São Paulo. Rev Paul Pediatr 2009;27:265-71.

29. Giugliano R, Carneiro EC. Factors associated with obesity in school children. J Pediatr (Rio J) 2004;80:17-22.

30. Guimarães LV, Barros MB, Martins MS, Duarte EC. Fatores associados ao sobrepeso em escolares. Rev Nutr 2006;19:5-17.

31. Corso AC, Buralli KO, Souza JM. Physical growth in schoolchildren from Florianópolis, Santa Catarina State, Brazil: a case-control study. Cad Saude Publica 2001;17:79-87.

32. Soar C, Vasconcelos FA, Assis MA, Grosseman S, Luna ME. Prevalence of overweight and obesity in school children in public school of Florianópolis, Santa Catarina. Rev Bras Saúde Matern Infant 2004;4:391-7.

33. Ricardo GD, Caldeira GV, Corso AC. Prevalence of overweight and obesity and central adiposity indexes among school-aged children in Santa Catarina, Brazil. Rev Bras Epidemiol 2009;12:424-35.

34. Ronque ER, Cyrino ES, Dórea VR, Serassuelo Jr H, Galdi EH, Arruda M. Prevalence of overweight and obesity in schoolchildren of high socioeconomic level in Londrina, Paraná, Brazil. Rev Nutr 2005;18:709-17.

35. Triches RM, Giugliani ER. Obesity, eating habits and nutritional knowledge among school children. Rev Saude Publica 2005;39:541-7.

36. Speiser PW, Rudolf MC, Anhalt H, Camacho-Hubner C, Chiarelli F, Eliakim A et al. Childhood obesity. J Clin Endocrinol Metab 2005;90:1871-87.

37. Dehghan M, Akhtar-Danesh N, Merchant AT. Childhood obesity, prevalence and prevention. Nutr J 2005;4:24.

38. Uauy R, Albala C, Kain J. Obesity trends in Latin America: transiting from under- to overweight. J Nutr 2001;131:893S-9S.

39. Valente $F$, Franceschini T, Burity V. A exigibilidade do direito humano à alimentação adequada. Ação Brasileira pelos Direitos Humanos e Nutrição. Brasilia: ABRANDH; 2007.

40. Pacto Internacional dos Direitos Econômicos, Sociais e Culturais [homepage on the Internet]. Assembleia Geral das Nações Unidas de 16 de dezembro de 1966. [cited 2010 Jan 22]. Available from: http://www.dhnet.org.br/direitos/sip/ onu/doc/pacto1.htm

41. Jew S, AbuMweis SS, Jones PJ. Evolution of the human diet: linking our ancestral diet to modern functional foods as a means of chronic disease prevention. J Med Food 2009;12:925-34.

42. Chaput JP, Tremblay A. Obesity and physical inactivity: the relevance of reconsidering the notion of sedentariness. Obes Facts 2009;2:249-54.

43. Dunstan DW, Barr ELM, Healy GN, Salmon J, Shaw JE, Balkau B, et al Television viewing time and mortality: the Australian Diabetes, Obesity and Lifestyle Study (AusDiab). Circulation 2010;121:384-91.

44. Brasil. Ministério da Saúde. Secretaria de Atenção à Saúde. Departamento de Atenção Básica. Obesidade: cadernos de atenção básica 12: série A. Brasília: Ministério da Saúde; 2006.

45. Santos LA. Educação alimentar e nutricional no contexto da promoção de práticas alimentares saudáveis. Rev Nutr 2005;18:681-92.

46. Silva MV. School meal programs as a means to meet nutritional requirements for students in the Integrated Public School Centers (CIEPS). Cad Saude Publica 1998;14:171-80

47. Davanço GM, Taddei JA, Gaglianone CP. Knowledge, attitudes and practices of teachers of basic cycle, exposed and non exposed to a Nutrition Education Course. Rev Nutr 2004;17:177-84.

48. Instituto Brasileiro de Geografia e Estatística - IBGE [homepage on the Internet]. Pesquisa Nacional de Saúde do Escolar, 2009 [cited 2009 Aug 29]. Available from: http://www.ibge.gov.br/home/presidencia/noticias/pense.shtm

49. Brasil. Ministério da Saúde. Secretaria de Atenção à Saúde. Departamento de Atenção Básica. Manual operacional para profissionais de saúde e educação: promoção da alimentação saudável nas escolas: série A. Brasília: Ministério da Saúde; 2008. 